\title{
Lichen Planus Pigmentosus Inversus: A Rare Subvariant of Lichen Planus Pigmentosus
}

\author{
Anne Guertler ${ }^{a} \quad$ Natalie Evenschor ${ }^{a} \quad$ Marlene Seegraeber $^{a}$ \\ Lars Einar French $^{a}$ b Véronique Weiler ${ }^{c} \quad$ Michael Flaig $^{a}$ \\ Daniela Hartmann ${ }^{\mathrm{a}}$ \\ aDepartment of Dermatology and Allergy, University Hospital of Munich, LMU, Munich, \\ Germany; ${ }^{b}$ Dr. Phillip Frost Department of Dermatology \& Cutaneous Surgery, University of \\ Miami, Miller School of Medicine, Miami, FL, USA; 'Dermpath München, Munich, Germany
}

\section{Keywords}

Lichen planus · Lichen planus pigmentosus

\begin{abstract}
Lichen planus pigmentosus inversus (LPPI) is a rare subvariant of Lichen planus pigmentosus (LPP), presenting with sharply defined brown to gray macules, papules, and plaques limited to the intertriginous areas, with only a few cases reported in the medical literature so far. While LPP mostly affects patients with Fitzpatrick skin type III-IV in sun-exposed areas such as the neck, LPPI is seen in Caucasians and spares sun-exposed areas. Skin lesions tend to be very refractory to treatment attempts including potent topical steroids and oral corticosteroids. Given the increased penetration of potent topical steroids and the high risk of skin atrophy, especially when applied to intertriginous areas, this case shows that topical calcineurin inhibitors (tacrolimus $0.1 \%$ ) might offer an effective and safe treatment option for LPPI.
\end{abstract}

\section{Introduction}

Lichen planus pigmentosus inversus (LPPI), first described in 2001 by Pock et al. [1], is a rare subvariant of Lichen planus pigmentosus (LPP) with only a few cases reported in medical literature so far. While LPP mostly affects patients with Fitzpatrick skin type III-IV (high prevalence in Asia, the Middle East, and South America) in sun-exposed areas such as the neck, LPPI is seen in Caucasians and spares sun-exposed areas. While an unilateral appearance has also been described [2], lesions are found bilaterally in most cases, affecting 
regions such as axillae, submammary folds, abdomen, groins, popliteal fossa, and lumbar region. Unlike lichen planus, LPPI lesions only cause mild to no pruritus. No abnormalities of the hair, the nails, or the mucous membranes have been reported. The etiology of LPPI remains unknown with the Koebner phenomenon due to mechanical stress of tight clothes as a potential trigger factor [3]. Spontaneous remission has been reported [4]. So far, skin lesions seem to be very refractory to treatment attempts including potent topical steroids and oral corticosteroids.

\section{Case Presentation}

A 76-year-old male patient with Fitzpatrick skin type II consulted the outpatient clinic of the Department of Dermatology and Allergy, University Hospital of Munich (LMU), Germany, showing bilateral pigmented axillary and inguinal lesions accompanied by mild pruritus. He reported that the lesions were persistent for 4 months (shown in Fig. 1a, b). Neither he nor his family had previously suffered from any skin conditions or allergies. Sun exposure prior to the occurrence of the skin lesions was denied. The patient was in an overall good health and nutritional status with a prehistory of persistent atrial fibrillation and arterial hypertension. Three years ago, he underwent unilateral mastectomy due to breast cancer. Thereafter, he had regular oncologic follow-up visits. Chemotherapy, radiation, or hormone therapy had not been administered. His long-term medication included bisoprolol, digoxin, and rivaroxaban.
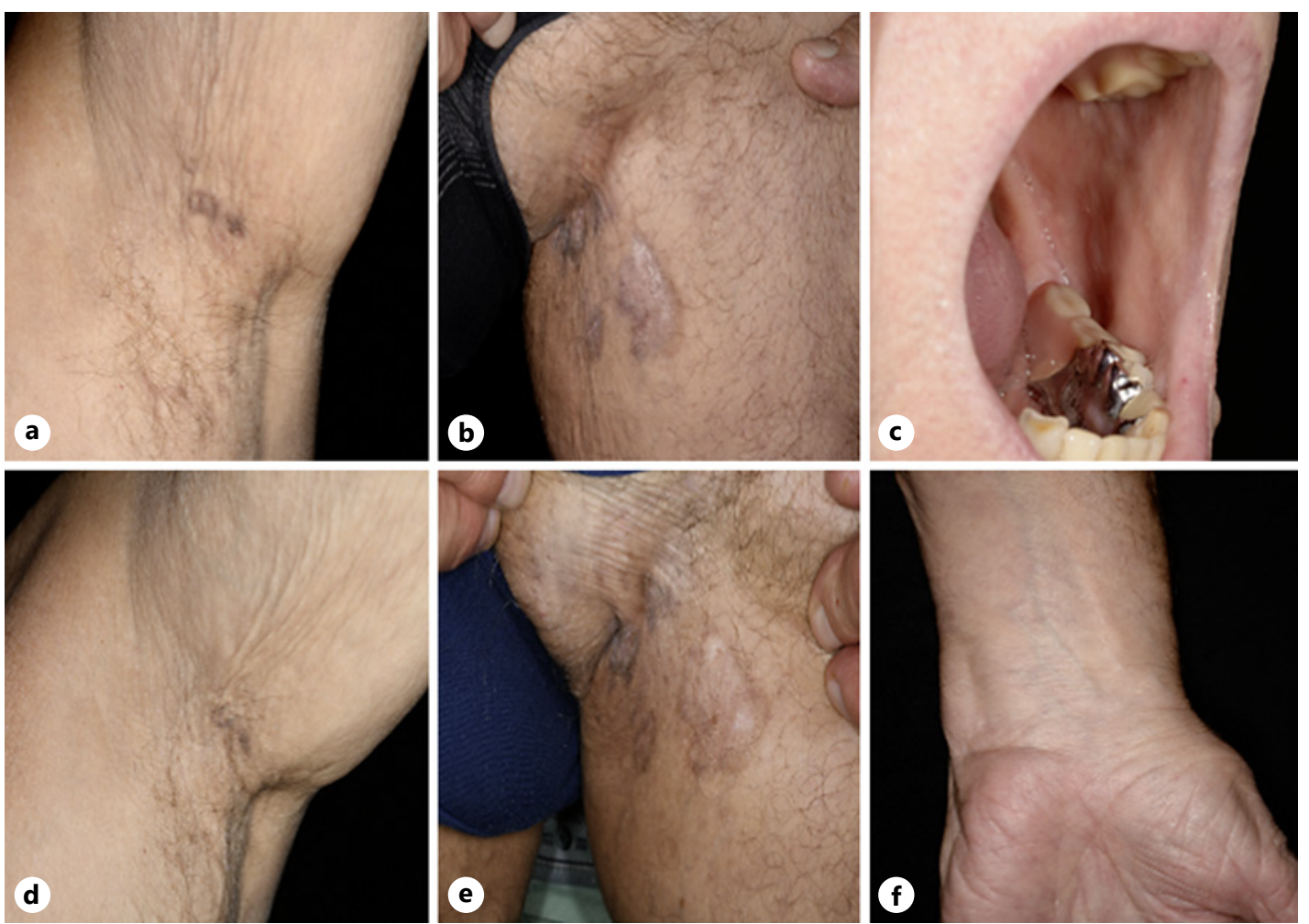

Fig. 1. Pigmented macules, papules, and plaques in the left axillary and left inguinal region before (a, b) and after 4 months of treatment with tacrolimus $0.1 \%$ once a day (d, e). Predilection sides of lichen planus including the oral mucous membrane (c) and the wrists (f) were not affected. 
The clinical examination showed sharply defined brown to gray macules, papules, and plaques limited to the intertriginous areas. Mucous membranes were free of pathology (shown in Fig. 1c). Differential diagnoses included fixed drug eruption, contact dermatitis with postinflammatory lesions, acanthosis nigricans, or erythema dyschromicum perstans. Punch biopsies taken from the intertriginous lesions revealed band-like lichenoid inflammatory infiltrates with epidermal atrophy, hypergranulosis, and discreet orthohyperkeratosis. The dermis showed an accumulation of macrophages containing pigments (shown in Fig. 2). The suspected clinical diagnosis of LPPI was confirmed. Predilection sites for lichen planus were not affected (shown in Fig. 1c, f). Routine laboratory tests, hepatitis serology, and a patch test performed showed no anomalies.

Topical tacrolimus $0.1 \%$ cream was prescribed as an off-label treatment once a day. Follow-up examinations were performed after 2 and 4 months. Over time, the intertriginous lesions became lighter and flatter, reduced in size, and symptoms of itching diminished (shown in Fig. 1d, e). No side effects were observed. We therefore suggest that topical tacrolimus $0.1 \%$ cream as a potential safe, effective, and simple therapeutic option for LPPI.

\section{Conclusion}

To our knowledge, this is the first published report describing a case of LPPI in Germany. Histopathological analysis allowed distinct diagnosis of LPPI by revealing epidermal atrophy, variable density of band-like lymphocyte infiltration, pigmentary incontinence, and melanophages in the dermis [5]. Given the increased penetration of potent topical steroids and the high risk of skin atrophy, especially when applied to intertriginous areas, topical calcineurin inhibitors such as tacrolimus $0.1 \%$ cream have a preferable low side effect profile and seem to be an effective treatment option for LPPI.

\section{Statement of Ethics}

The research was conducted ethically in accordance with the World Medical Association Declaration of Helsinki. Written informed consent was obtained from the patient for publication of this case report and any accompanying images.

Fig. 2. Histopathological finding (hematoxylin and eosin stain) with epidermal atrophy, hypergranulosis, discreet orthohyperkeratosis with band-like lichenoid inflammatory infiltrates, and melanophages in the dermis.

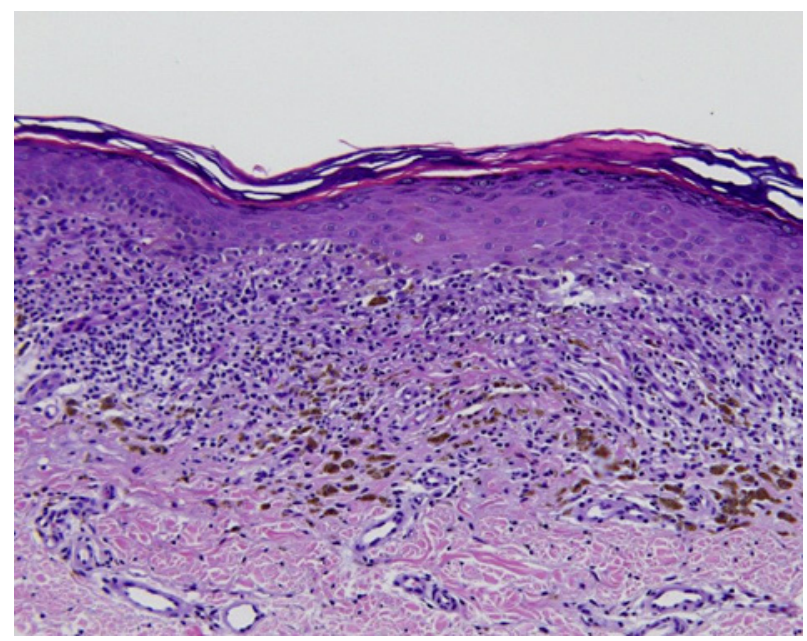




\section{Conflict of Interest Statement}

The authors have no conflicts of interest to declare.

\section{Funding Sources}

The authors did not receive any funding.

\section{Author Contributions}

Anne Guertler: conceptualization, data curation, investigation, methodology, visualization, writing of the original draft, writing, review, and editing. Natalie Evenschor: conceptualization, data curation, and editing. Marlene Seegraeber: conceptualization, data curation, and editing. Lars Einar French: supervision, validation and editing. Véronique Weiler: methodology and validation. Michael Flaig: supervision, methodology, and validation. Daniela Hartmann: supervision, methodology, validation, writing, and editing.

\section{References}

1 Pock L, Jelinkova L, Drlik L, Abrhamova S, Vojtechovska S, Sezemska D, et al. Lichen planus pigmentosusinversus. J Eur Acad Dermatol Venereol. 2001;15(5):452-4.

2 Patel K, Arballo 0, Wohltmann W. Unilateral hyperpigmented flexural lesion in the left axilla. JAAD Case Rep. 2020;6(2):131-2.

3 Majima Y, Yagi H, Ikeda Y, Ito T, Tokura Y. Two cases of lichen planus pigmentosus inversus: possible causative role of tightly fitting underclothes. Eur J Dermatol. 2013;23(6):904-5.

4 Ryynanen AT, Von Willebrand M, Kluger N. Lichen planus pigmentosus-inversus in a Finnish man. J Eur Acad Dermatol Venereol. 2019;33(2):e64-5.

5 Gaertner E, Elstein W. Lichen planus pigmentosus-inversus: case report and review of an unusual entity. Dermatol Online J. 2012;18(2):11. 